\title{
The Hard X-ray Nanoprobe Beamline at Diamond - Current Status
}

$\underline{\text { Paul Quinn }}^{1 *}$, Julia Parker ${ }^{1}$, Fernando Cacho-Nerin ${ }^{1}$, Mike Walker $^{2}$ and Paul Howes ${ }^{2}$

${ }^{1}$. Diamond Light Source, Harwell Science and Innovation Campus, Didcot, OX11 ODE, UK

2. University of Leicester, Department of Physics, Leicester, LE1 7RH, UK

*Corresponding author, paul.quinn@ diamond.ac.uk

\section{Introduction}

The hard X-ray nanoprobe beamline at Diamond is a new facility for nanoscale microscopy. The beamline operates over a 4.5 - to $23-\mathrm{keV}$ energy range with an emphasis on multi-modal analysis providing elemental mapping, speciation mapping by XANES, structural phase mapping by nano-XRD and imaging through differential phase contrast and ptychography. A flexible scanning system allows for fast acquisition and arbitrary scan paths, simple data acquisition software and the ability to process data in near real-time. I14 welcomed its first users for commissioning experiments in March 2017 and completed its first user run between Oct 2017 and Mar 2018. The beamline is in an optimization phase with new techniques and users tools rolling out over a two year ramp up period.

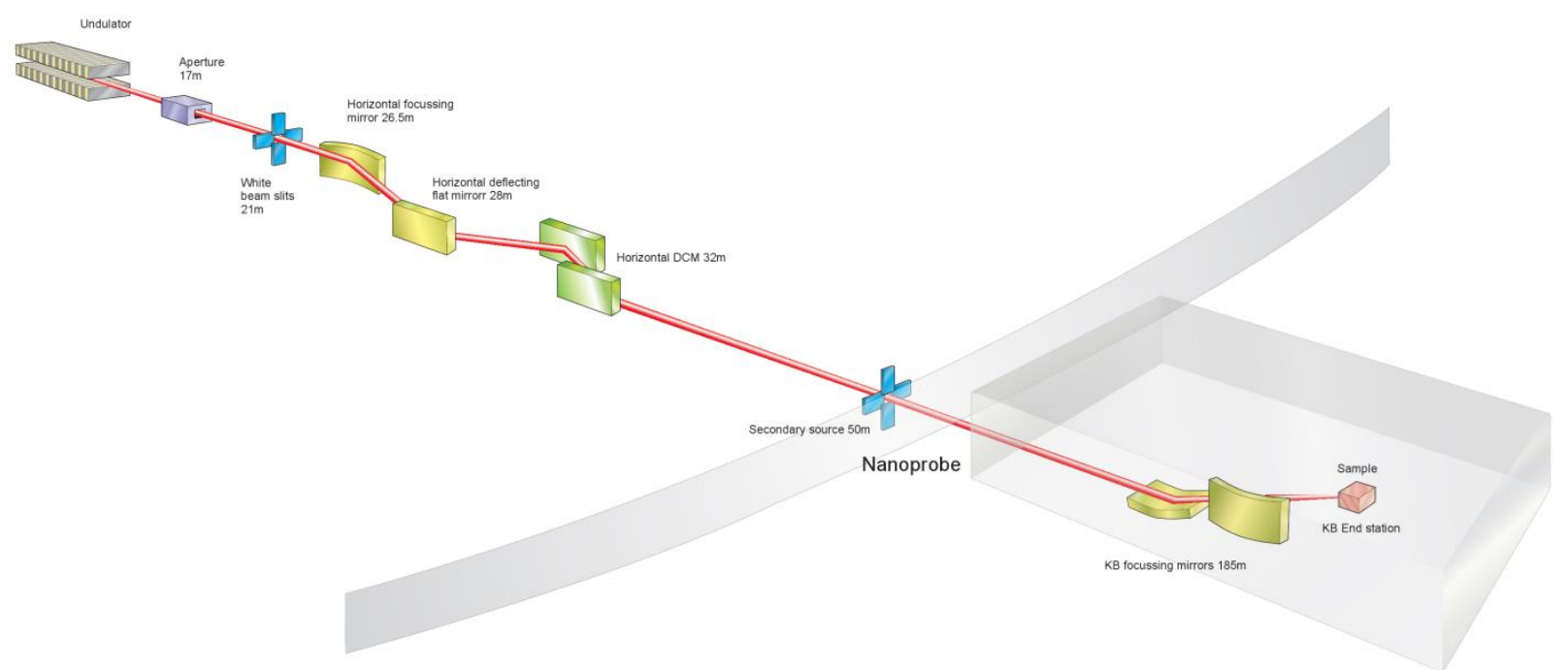

Figure 1. Schematic of beamline I14

\section{Characteristics and status of the beamline}

The I14 beamline is $186 \mathrm{~m}$ long. The length of the beamline is to improve demagnification of the beam at the sample while maintaining a reasonable working distance and to provide a coherent beam for diffraction limited focussing and coherent imaging applications. The beamline uses an all horizontally deflecting geometry (Figure 1). Mirrors M1 (with bender) \& M2 (flat) use stripes of Si, Rh and Pt to provide harmonic rejection and horizontal beam shaping with respect to the secondary source. The 
horizontally deflecting monochromator is a fixed exit system using a pair of $\mathrm{Si}(111)$ crystals. The beam propagates to an external building before it is finally focused using pre-shaped $\mathrm{KB}$ mirrors.

\section{Example results - Microstructural study of cold spray coatings}

One of the first experiments on I14 was a study of thermal spray coatings. Thermal spray is a process of depositing metallic powders onto a solid substrate material to form a coating. The particles have diameters ranging from 5 to $40 \mu \mathrm{m}$ and are accelerated using a converging-diverging deLaval nozzle and a high pressure $(10-60$ bar $)$, pre-heated $\left(300-1100^{\circ} \mathrm{C}\right)$ inert gas jet, which usually consists of helium (He) or nitrogen (N2) [1]. The inert gas rapidly cools as it expands at the nozzle exit, thus the particles do not achieve melting point and the deposition occurs at high velocities (300 to $1200 \mathrm{~m} / \mathrm{s}$ ) and kinetic energies, so the solid particles are plastically deformed on impact, producing bonded splats on the surface. Beamline I14 was used probe local variations across a splat particle. XRD patterns from the top and near inter-facial region of a deposited particle are

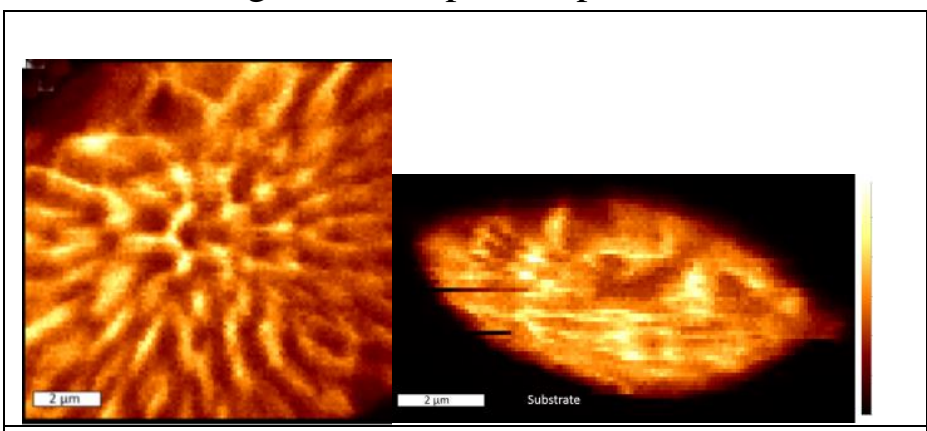

Figure 3. Left an XRF map of $\mathrm{Nb}$ in a power particle of $\mathrm{Ni}$ alloy 718. Right. Map from a deposited particle. Sample extracted via FIB milling and thinned to $1 \mu \mathrm{m}$

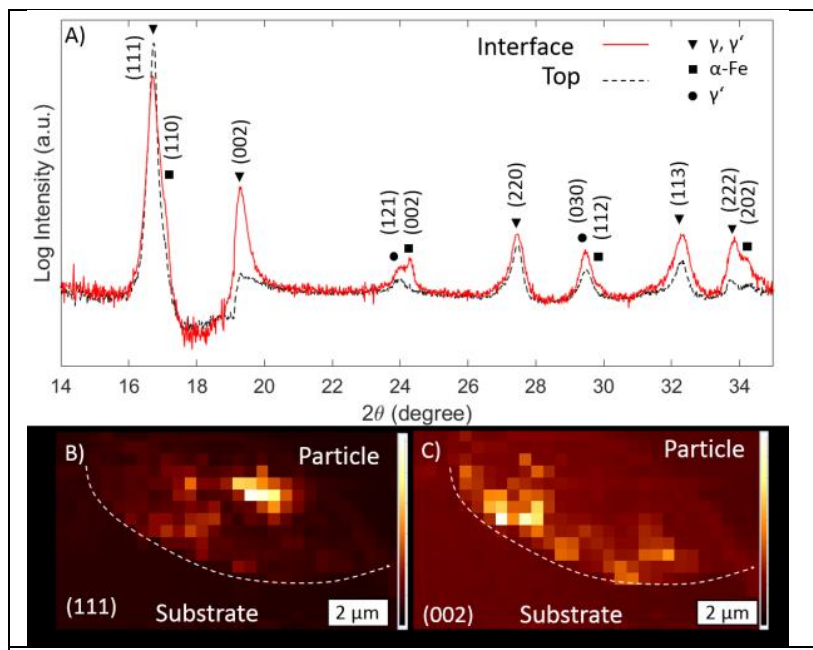

Figure 2 A) Diffraction patterns comparing the difference in intensities of the (111) and (002) directions at different regions of the particle. Intensity maps for the B) (111) and C) (002).

compared in Figure 2. The (111) reflection is stronger at the top of the splat, whereas the (002) reflection is more intense towards the interface (Fig. 2A). A map of the intensity of the reflection, Fig. 2B and Fig. 2C, for the (111) and (002) directions may suggest that local strain and deformation at the interface leads to preferential re-crystallisation and alignment of the crystal in certain directions[2]. The XRF maps also show that the $\mathrm{Nb}$ segregation found in the raw powder particle is maintained, however the structure is less defined and more distorted compared to the un-sprayed powder.

\section{Future outlook}

The beamline has completed a number of successful experiments and the focus is now on streamlining the analysis and rolling out new techniques in particular ptychography and cryogenic sample handling.

\section{References}

[1] Villafuerte, J., Modern Cold Spray - Materials, process and Application, Springer, 1st edn. (2015). [2] Zahiri, S. H. et al., "Recrystallization of cold spray-fabricated CP titanium structures", Journal of Thermal Spray Technology, Vol. 18, No. 1, (2008) pp. 16-22 